\title{
What are the degrees of freedom in the partonic fluid at RHIC?
}

\author{
Rene Bellwied \\ Physics, Department, Wayne State University, \\ Detroit, MI 48202, U.S.A. \\ E-mail: bellwiedephysics.wayne.edu
}

\begin{abstract}
Recent RHIC data show evidence of multiple hadron production mechanisms in heavy ion collisions compared to simple fragmentation in vacuum. I will review the measurements of collective flow, high momentum quenching, and two particle angular correlations to show that neither thermal production nor string fragmentation can describe the abundances, the angular distributions or the kinematic properties of all hadrons produced at RHIC. The proposed new hadronization mechanisms not only serve as evidence for a deconfined partonic phase of matter, but also for strong coupling of the degrees of freedom in the deconfined phase. I will point out a surprising lack of flavor dependence in these properties at RHIC, though, which might have to lead to further revisions of our understanding of the relevant degrees of freedom in the partonic phase and during the hadronization process.
\end{abstract}

Critical Point and Onset of Deconfinement 4th International Workshop

July 9-13 2007

GSI Darmstadt,Germany 


\section{Introduction}

The measurements of anisotropic particle flow and jet quenching at RHIC have revealed a deconfined state of matter at high temperature and partonic density, which is characterized best as a near perfect fluid, i.e. a collective state with an extremely low ratio of shear viscosity to entropy. Indeed over the past year the experiments at RHIC were able to experimentally verify the original conjecture of a state near the quantum limit through several independent measurements of the $\eta / \mathrm{s}$ ratio. Fig. 1 shows a summary of calculations based on $\left\langle\mathrm{p}_{T}>\right.$-fluctuation, light and heavy quark elliptic flow, and quenching measurements [1, 2, 3, 田. These calculations are still model dependent, but it is intriguing to recognize that, if the initial conditions assumed in the models are correct, the new state of matter is not well described by either perturbative QCD or a purely hadronic model.

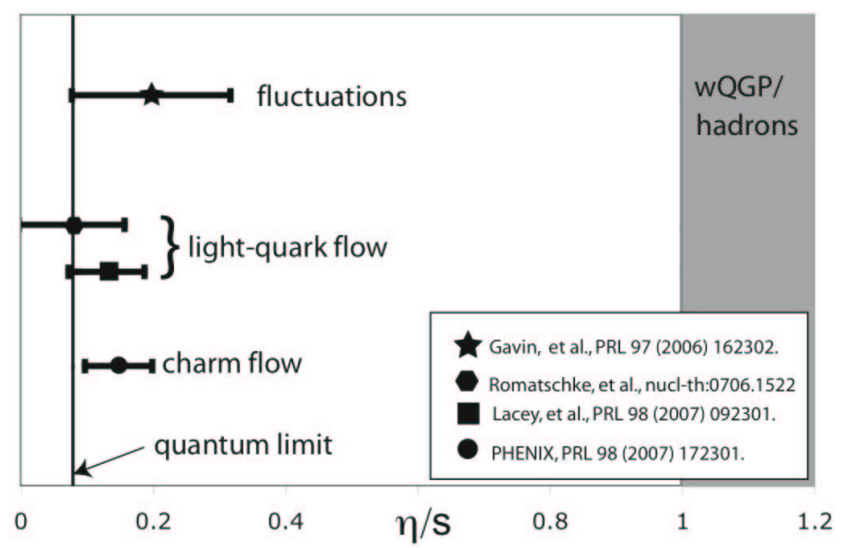

Figure 1: Summary of recent model dependent determinations of the shear viscosity over entropy ratio $(\eta / \mathrm{s})$ based on measurements from STAR and PHENIX.

This led to the definition of the sQGP, a strongly coupled Quark Gluon Plasma, rather than the weak coupling phase we expected from lattice QCD at sufficiently high initial temperature. Since then recent lattice calculations and their comparison to hard thermal loop calculations have revealed that the conditions at RHIC are not sufficient to reach the weakly coupled limit, but that the LHC energies might be sufficient to generate perturbative QCD like conditions [5]. This unexpected behavior of the deconfined matter at RHIC poses the question of the nature of the degrees of freedom in the partonic liquid and the details of the QCD phase transition from a strongly coupled partonic system to the hadronic gas. Clearly this mechanism might be different to the fragmentation picture in vacuum which has been verified through numerous measurements in elementary particle collisions over the past three decades. In this paper I will try to show that the particle emission spectra, the particle correlations as well as the anisotropic particle flow reveals details of competing hadronization mechanisms in heavy ion collisions which are either non-existent or much less prominent in elementary collisions. These novel mechanisms might have a profound impact on our understanding of the formation of baryonic matter in the universe.

\section{The difference between hadronization in vacuum and in medium}

In 1977 two competing papers appeared that tried to model the hadronization process in high 
energy elementary collisions. The initial Feynman/Fields paper describes the hadronization process in vacuum through jet fragmentation [6]. This approach was later on extended to string fragmentation. Although no explicit hadronization mechanism is given in this picture the hadronic particle distribution can be parametrized through the fragmentation function $\mathrm{D}_{q}^{h}$, which yields the probability that a certain parton ' $q$ ' fragments into a certain hadron ' $h$ '. Baryon formation in such a model generally requires the formation of a di-quark structure, as a remnant of the initial hard scattering in a proton-proton collision.

The other paper was by Das and Hwa [7] and it tried to describe hadronization through recombination or coalescence of independent free quarks. The clustering of quarks is modeled through a momentum overlap probability function. Again, there is no explicit hadronization mechanism, but the particle emission spectra are described well by this approach. This model has been the basis for many recent recombination models used in heavy ion collisions (e.g. [8, 9]).

Over the years the fragmentation approach has been widely accepted as the main hadronization mechanism in vacuum, but recent evidence in particle spectra measured in heavy ion collisions at RHIC has re-ignited the interest in the recombination approach.

This is mainly due to two key heavy ion results, the particle identified elliptic flow and quenching measurements. In both cases the intermediate transverse momentum region of the measured spectra has revealed a scaling, the so-called constituent quark scaling, which can be interpreted as evidence for not only deconfinement but also quark recombination. Figs. 2 and 3 show a summary of the results for $\mathrm{R}_{C P}$, the nuclear suppression factor defined as the ratio of the transverse momentum spectra measured in different centrality bins and scaled with the appropriate number of binary collisions, and v2, the second moment of the Fourier decomposition of the measured identified momentum spectra.

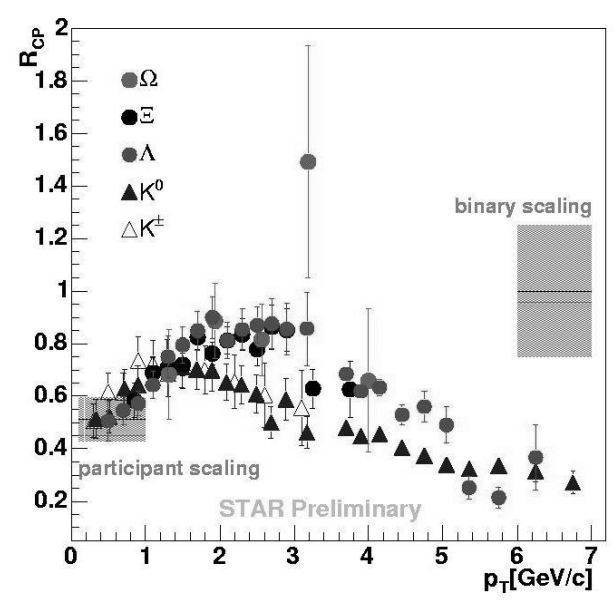

(a)

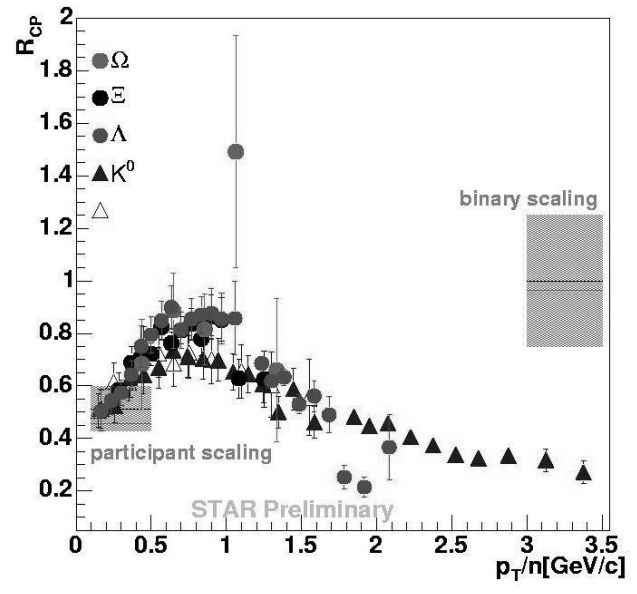

(b)

Figure 2: STAR data on (a) $\mathrm{R}_{C P}$ vs $\mathrm{p}_{T}$ in $\mathrm{Au}+\mathrm{Au}$ Collisions at $\sqrt{s_{N N}}=200 \mathrm{GeV}$. (b) $\mathrm{R}_{C P} \mathrm{vs} \mathrm{p}_{T} / \mathrm{n}$ in $\mathrm{Au}+\mathrm{Au}$ collisions at $\sqrt{s_{N N}}=200 \mathrm{GeV}$ using $\mathrm{n}=3$ for baryons and $\mathrm{n}=2$ for mesons. $\mathrm{R}_{C P}$ is calculated from $0-5 \%$ and 40-60\% central $\mathrm{Au}+\mathrm{Au}$ collisions.

The scaling parameter could be called the number of constituent quarks or the number of 


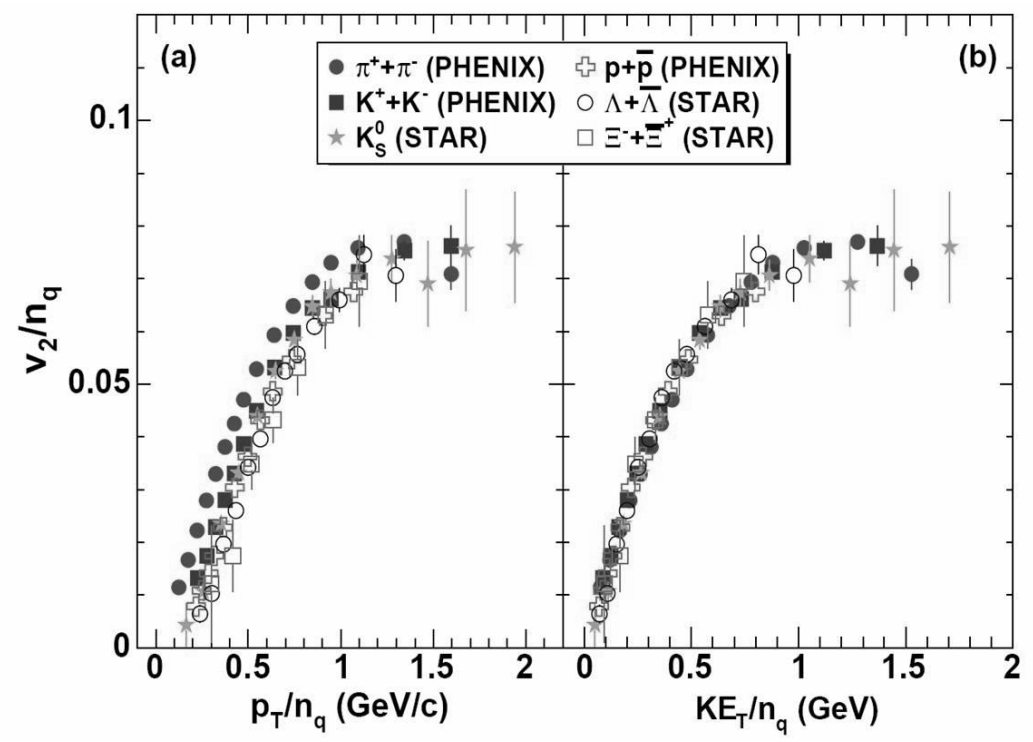

Figure 3: $v 2 / \mathrm{n}_{q}$ vs. $\mathrm{p}_{T} / \mathrm{n}_{q}$ and $\mathrm{KE}_{T} / \mathrm{n}_{q}$ for several particle species measured by STAR and PHENIX as indicated in $\mathrm{Au}+\mathrm{Au}$ collisions at $\sqrt{s_{N N}}=200 \mathrm{GeV}$ [10].
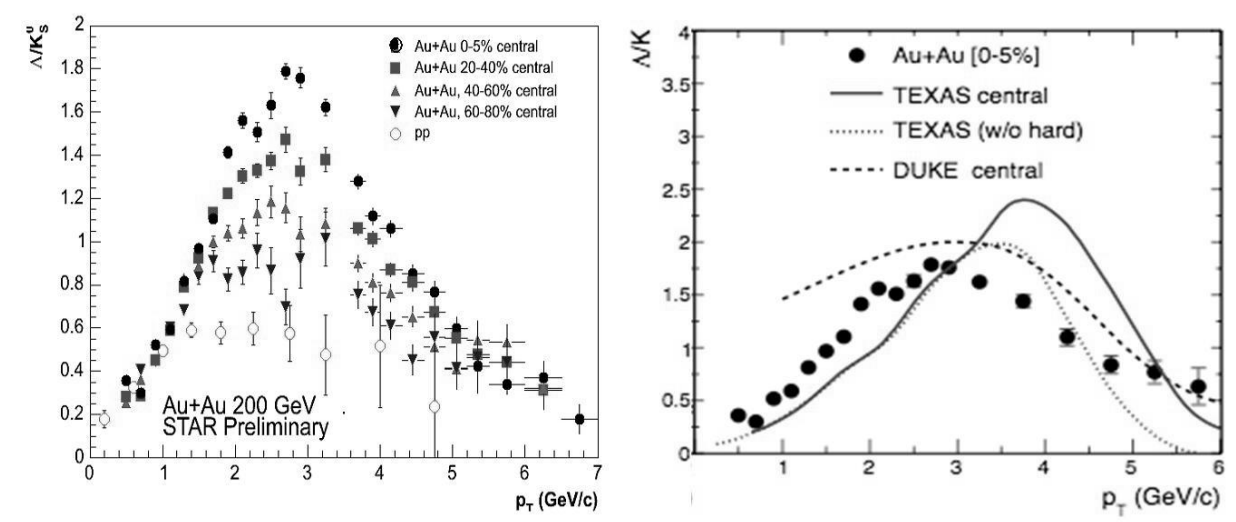

Figure 4: a.) $\Lambda / \mathrm{K}_{s}^{0}$ measurements in pp and AA collisions at different centralities as measured by STAR. b.) Comparison of central $\Lambda / \mathrm{K}_{s}^{0}$ to recombination models [8, 9].

valence quarks. Quark scaling apparently works, but the relevant degree of freedom is not well defined. Popular recombination models [8, 9] have taken the approach of using thermalized constituent quarks, with a well defined mass, to describe not only the flow data but also the unexpected baryon to meson $(\mathrm{B} / \mathrm{M})$ ratio at intermediate $\mathrm{p}_{T}$. A comparison to STAR $\mathrm{B} / \mathrm{M}$ measurements is shown in Fig.4.

The main features of the central data results are rather well described by the models. The B/M ratio peaks at about $2-3 \mathrm{GeV} / \mathrm{c}$ and it is considerably higher than in proton proton collisions. It is 
interesting to note, though, that the pp data already show a 'hump' at intermediate $\mathrm{p}_{T}$. This hump is rather well described by a tuned PYTHIA fit [11], which shows that string fragmentation can describe the overall trend of the baryon to meson production, but in terms of the amplitude of the $\mathrm{B} / \mathrm{M}$ ratio any fragmentation model underestimates the enhanced baryon production by at least a factor 2-3. Recombination on the other hand seems to be easily tuneable to the proper B/M ratio. The main reason is that in a thermalized fireball it is easy to achieve large momentum overlap of the quarks in the thermal pool, which then leads to the production of higher momentum baryons, whereas the fragmentation of very high momentum quarks into intermediate $\mathrm{p}_{T}$ baryons is a rare process. The required thermal pool of quarks is not achievable in elementary collisions, but likely in heavy ion collisions.

A direct comparison of the spectral features obtained in pp and AA collisions seems to further confirm the change in hadronization mechanism, at least for hadrons in a specific momentum range. The proton-proton measurements at RHIC reveal a breakdown of the so-called $\mathrm{m}_{T}$-scaling at intermediate $\mathrm{p}_{T}$ [16]. Instead of a common scaling for all identified $\mathrm{m}_{T}$ spectra, which was established in ISR measurements at lower collision energies, the RHIC data show a baryon/meson scaling at sufficiently high transverse momentum. This can be explained by requiring di-quark formation for baryon production, which leads to a di-quark suppression factor which needs to be applied to the baryon spectra in order to find a common hadron scaling. This effect is well described by the gluon fragmentation model in PYTHIA [11]. It is the first experimental evidence for di-quark formation at RHIC, though. Di-quark formation leads to baryon/meson differences but it can not describe the constituent quark scaling measured in AA collisions. In fact di-quark formation should lead to a distinct lack of scaling inasmuch as a diquark-quark based formation process should scale similar to the quark-antiquark based process. Investigations of scaling of balance functions for identified particles in pp are underway to test this hypothesis. The validity of the di-quark picture to describe the $\mathrm{m}_{T}$-scaling of the identified spectra in pp collisions requires gluon dominance in the fragmentation process at RHIC energies. Besides the $\mathrm{m}_{T}$ scaling, the lack of discernible differences in the particle vs. anti-particle production over the kinematic range measured at RHIC, and the enhanced gluon fragmentation contribution in PYTHIA and fragmentation function fits [17], necessary to describe RHIC data [16, 18], shows that at these collision energies the parton interactions are indeed dominated by low $\mathrm{x}$ gluons. This dominance is likely to further increase at the LHC due to the even lower $\mathrm{x}$ coverage at the higher energies.

Extensions to the simple inclusive B/M ratio measurements in heavy ion collisions have been recently performed by the STAR collaboration. B/M ratios were measured in structures which appeared in high momentum two-particle correlation measurements. Fig.5a shows a comparison of $\mathrm{B} / \mathrm{M}$ ratios in same-side and away-side jet cones triggered by a high momentum charged particle [12], Fig.5b shows a comparison of B/M ratios in the same side jet cone and the same-side long-range correlation ridge as measured by STAR [13]. In both cases it seems that inside the unquenched jet the $\mathrm{B} / \mathrm{M}$ ratio is consistent with expectations from fragmentation models, whereas the in-medium response to the traversing jets leads to a ratio that is better described by the recombination scenario.

At higher momenta $\left(\mathrm{p}_{T}>6 \mathrm{GeV} / \mathrm{c}\right)$ the particle identified spectra exhibit all the features of pure jet fragmentation, both in single particle and particle correlation measurements [14, 15]. 

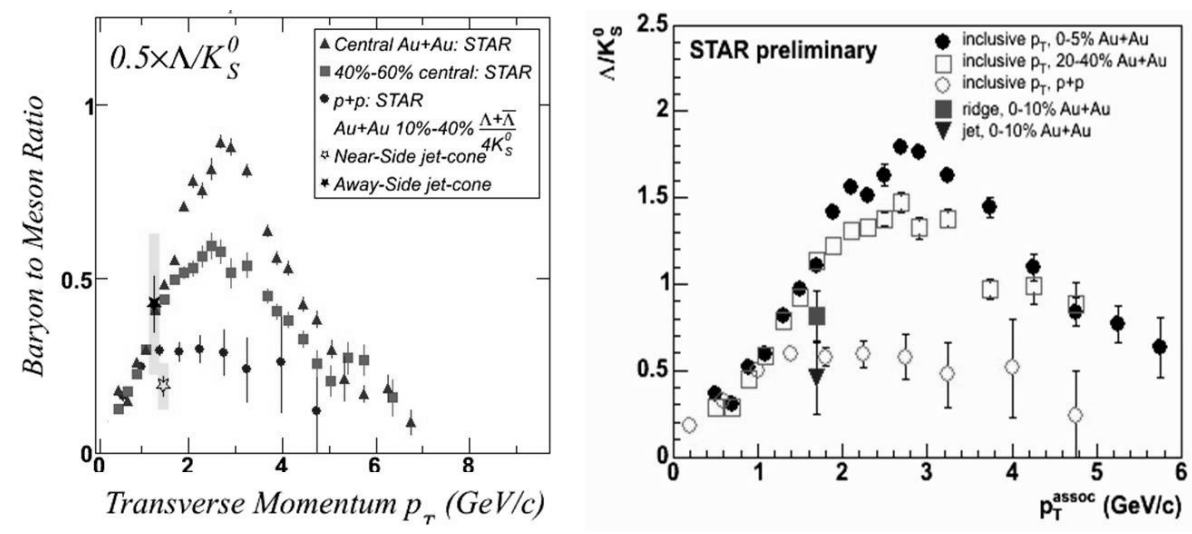

Figure 5: STAR measurements of the inclusive $\Lambda / K_{s}^{0}$ ratio as a function of centrality and transverse momentum, a.) compared to ratios measured in the same-side and away-side jet region from triggered two-particle correlations [12], b.) compared to ratios measured in the same-side jet and the long-range correlation ridge region from triggered two-particle correlations [13].

\section{Does strong coupling require a special degree of freedom ?}

It is interesting to note that there is a total lack of constituent quark mass dependence in the scaling of v2 as shown in Fig.3. In recombination approaches this is largely attributed to the fact that the input constituent quark mass of the up, down and strange quarks is quite similar (300 and $460 \mathrm{MeV}$, respectively) and that all identified particles measured until recently did not include heavier flavors. The recent measurement of the nuclear suppression factor and the elliptic flow for D-mesons, based on electrons from the semi-leptonic decay of the heavy mesons [19, 20, 21], allows us to determine the applicability of partonic recombination a little further, and early results seem to indicate that both, the $\mathrm{R}_{A A}$ and the $\mathrm{v} 2$ measurements, can only be explained if one assumes identical $\mathrm{p}_{T}$-dependencies for the flow and the quenching of light and heavy quarks as is shown in Fig. 6 for $\mathrm{R}_{A A}$ [22] and in Fig.7 for v2 [10].

At some high momentum the mass of the bare or constituent heavy quark should be negligible, but this should not be the case for the intermediate momenta measured here. Many models, as shown in Fig.4, have been proposed to address these measurements and in particular the apparent lack of a dead cone effect for induced gluon radiation, as well as the lack of a heavy quark mass dependence in the v2. The most successful of these models try to give the heavy quark a special status, by postulating either the survival of heavy quark resonant states above $\mathrm{T}_{c}$ [23, 24] or the reduced formation time of heavy quark hadrons from the partonic phase [25]. Fig.8 shows a comparison of the data to the heavy quark bound state model.

The near identical $\mathrm{p}_{T}$-dependence of the $\mathrm{v} 2$ and the quark energy loss between light and heavy quarks is very striking, though, and might require a much more fundamental explanation. One possibility is that the quasi-particle state formed near $\mathrm{T}_{c}$ is really not depending on the constituent or even bare quark mass concept, but rather simply the number of partons, which could be mostly gluons, until close to hadronization. Still, for a dynamic evolution measure such as the v2 as a 


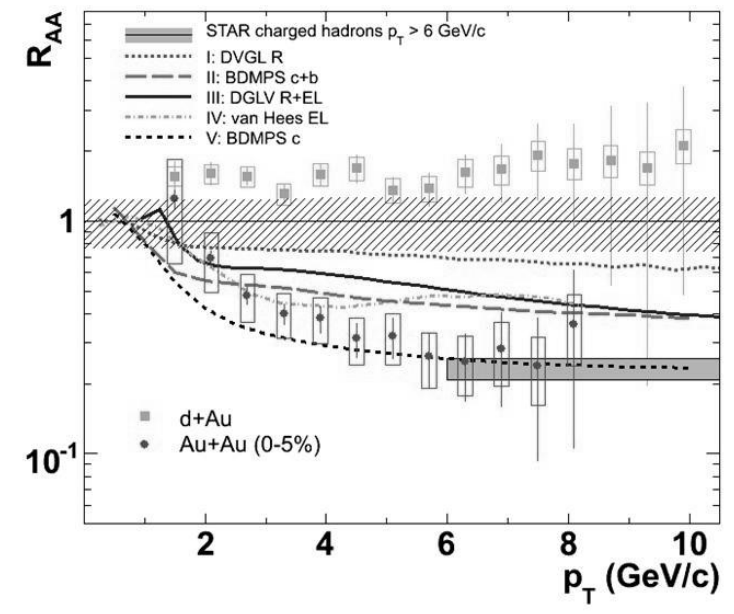

Figure 6: Nuclear suppression factor for non-photonic single electron spectra in semi-central Au+Au collisions at RHIC compared to the $\mathrm{R}_{A A}$ for charged hadrons (i.e. light quark suppression) and various models [22].

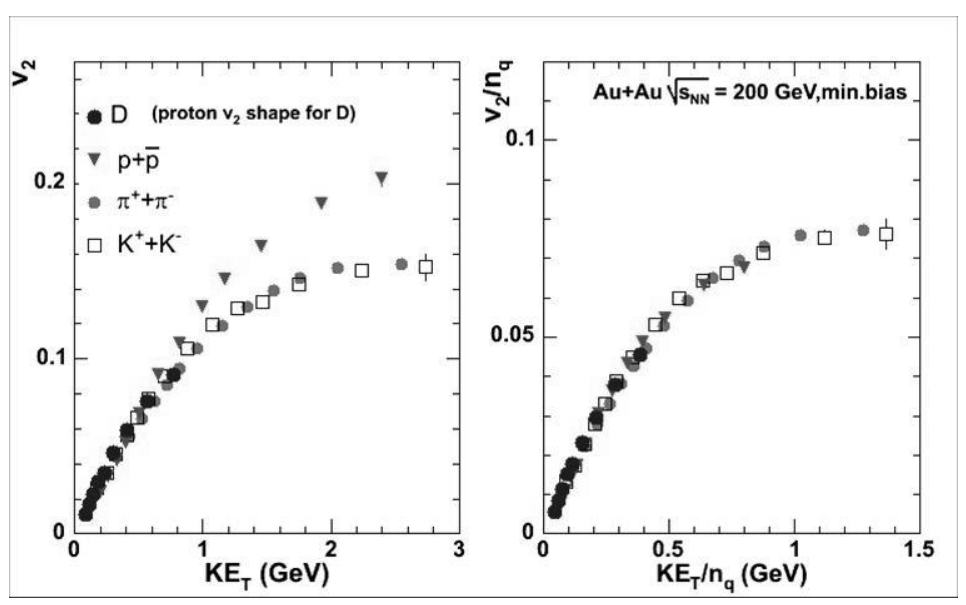

Figure 7: Elliptic flow (non-scaled and $\mathrm{n}_{q}$ scaled) for non-photonic single electron spectra in semi-central $\mathrm{Au}+\mathrm{Au}$ collisions at RHIC compared to light quark hadrons [10].

function of $\mathrm{p}_{T}$, the dynamics of the degree of freedom has to play a role, and at least the effect of the bare quark mass should be measurable if we indeed probe the fragmentation or recombination of quarks. A detailed measurement of reconstructed D-mesons and B-mesons is sorely needed to remove the ambiguities in the semi-leptonic measurements, and future measurements of high momentum heavy flavor mesons and baryons should answer the question whether the liquid phase above the critical temperature requires indeed a special degree of freedom to describe all features of hadronization from a dense deconfined medium. 

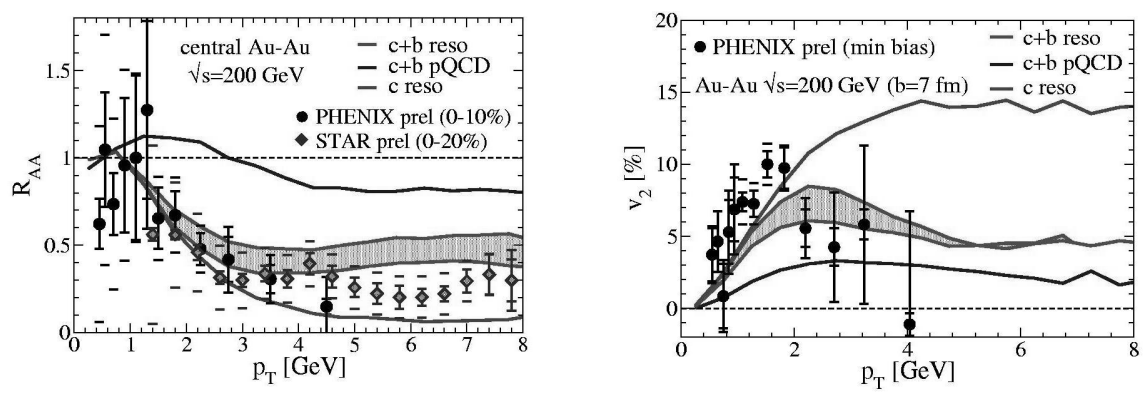

Figure 8: Nuclear suppression factor (left panel) and elliptic flow (right panel) for non-photonic single electron spectra in semi-central Au+Au collisions at RHIC. Data [19, 20, 21] are compared to theory predictions [24] using Langevin simulations with elastic c- and b-quark interactions in an expanding QGP fireball and heavy-light quark coalescence at hadronization [23].

\section{The relevance of RHIC results to the FAIR program}

Besides the very detailed and strong evidence for deconfinement at RHIC energies, the data reveal a surprising lack of evidence for chiral symmetry restoration. Vector meson and resonance measurements have been performed to new levels of precision at RHIC, in particular in the sector of heavy hadronic resonances, but the measurements are mostly used to determine the lifetime of the produced partonic and hadronic systems through detailed mapping of re-interaction probabilities [26]. In these measurements the properties of the resonances, such as mass, width, and branching ratios are generally in very good agreement with the particle data group references. There are small variations in mass and width for certain resonances as a function of their momentum, but they have been measured consistently in pp, dA, and AA collisions [27] and therefore should not be attributed to chiral symmetry restoration. Recently PHENIX has shown results that might indicate behavior similar to the NA45 and NA60 low mass di-lepton measurements [28], but whether this is evidence for medium modification of vector mesons remains to be seen. It seems that either our measurements are not sensitive to chiral symmetry restoration or that the chiral transition might indeed decouple from deconfinement, which is in disagreement with lattice QCD calculations. A very high luminosity program at FAIR should enable more detailed measurements of medium modification, in particular for chiral partners in the heavy quark sector. It is remarkable to realize that although open charm production is at threshold at FAIR energies, the yield of open charm obtained in a 25 week run at CBM is about an order magnitude larger than the yield STAR obtains over the same period of time [29]. Detailed measurements, not only of chiral partners, but also particle identified elliptic flow, radial flow and jet quenching might therefore be possible albeit at a slightly lower $\mathrm{p}_{T}$-range. The main purpose of these measurements should be to map out the disappearance of the strong sQGP signatures, such as quark scaling, hydro scaling, and high $\mathrm{p}_{T}$ suppression. These results will complement the thrust of the CBM program, which emphasizes the search for a critical point in the QCD phase diagram. 


\section{References}

[1] S. Gavin and M. Abdel-Aziz, Phys. Rev. Lett. 97, 162302 (2006)

[2] P. Romatschke and U. Romatschke, arXiv:0706.1522

[3] R. Lacey et al., Phys. Rev. Lett. 98, 092301 (2007)

[4] A. Adare et al. (PHENIX coll.), Phys. Rev. Lett. 98, 172301 (2007)

[5] J.P. Blaizot et al., hep-ph/0611393

[6] R.D. Field and R.P.Feynman, Phys. Rev. D15, 2590 (1977)

[7] K.P. Das And R.C. Hwa, Phys. Lett. B68, 459 (1977)

[8] R.J. Fries et al. Phys. Rev. Lett. 90, 202303 (2003).

[9] V. Greco et al., Phys. Rev. Lett. 90, 202302 (2003)

[10] see e.g. A.Taranenko for PHENIX collaboration, nucl-ex/0703025

[11] M. Heinz, contribution to Hot Quarks 2006

[12] J. Zuo, arXiv:0705.3286

[13] J. Bielcikova, arXiv:0707.3100

[14] J. Adams et al. (STAR coll.), Phys. Rev. Lett. 91172302 (2003)

[15] K. Adcox et al. (PHENIX coll.), Phys. Rev. Lett. 88, 022301 (2002)

[16] B. Abelev et al. (STAR coll.), Phys. Rev. C75, 064901 (2007)

[17] S. Albino et al., hep-ph/0502188

[18] B. Abelev et al. (STAR coll.), Phys. Lett. B637, 161 (2006)

[19] S.S. Adler et al. (PHENIX coll.), Phys.Rev.Lett. 94, 082301 (2005)

[20] J.Bielcik for STAR collaboration, Nucl.Phys. A774, 697 (2006)

[21] S.S. Adler et al., PHENIX collaboration, Phys.Rev. C72, 024901 (2005)

[22] B. Abelev et al., STAR coll., Phys.Rev.Lett. 98, 192301 (2007)

[23] R. Rapp and H. Van Hees, J.Phys. G32, S351 (2006)

[24] H. van Hees et al., Phys. Rev. C73, 034913 (2006)

[25] A. Adil and I. Vitev, Phys.Lett. B649, 139 (2007)

[26] B. Abelev et al. (STAR coll.), Phys. Rev. Lett. 97, 132301 (2006)

[27] J. Adams et al. (STAR coll.), Phys. Rev. Lett. 92, 092301 (2004)

[28] S. Afanasiev et al. (PHENIX coll.), arXiv:0706.3034

[29] P. Senger, contribution at BORMIO 2007 